\title{
LA PROGRAMACIÓN EN EL ESCENARIO CONVERGENTE: OFERTA MEDIÁTICA DE RADIO Y TELEVISIÓN
}

Jéssica Izquierdo-Castillo': Universitat Jaume I. España jizquier@com.uji.es

\section{RESUMEN}

En los estudios de grado de Comunicación Audiovisual se recoge el estudio de la materia sobre fundamentos de la radio y la televisión. En este contexto, destaca el análisis sobre la oferta programática de los medios televisivos y radiofónico, especialmente en su vinculación con la convergencia mediática. Los conocimientos y competencias adquiridos por el alumnado de estos estudios en materia de programación de los medios audioviusales con mayor audiencia repercute en una formación más completa y una mejor preparación para el posterior desempeño profesional y/o académico. La televisión y la radio ocupan una posición central en el sistema comunicativo, debido principalmente a la acumulación de audiencia y su consiguiente repercusión sobre la esfera pública. En este sentido, ambos medios requieren un estudio detenido que recorra su naturaleza mediática, sus estrategias programáticas y su desarrollo hacia la convergencia. Además, resulta imprescindible conocer los mecanismos y formas adoptadas por los actores involucrados, ya sean entes que operan en territorio nacional como casos de mercados externos al sistema comunicativo español, para conocer cuál es su ubicación en el contexto convergente.

PALABRAS CLAVE: Programación - Radio - Televisión - Convergencia - Grado

\footnotetext{
${ }^{1}$ Autor correspondiente

Jéssica Izquierdo Castillo: Universitat Jaume I. Castellón. España

Correo: jizquier@com.uji.es
} 


\title{
PROGRAMS INTO CONVERGENCE SCENARIO: RADIO AND TELEVISION MEDIA OFFER
}

\begin{abstract}
Audiovisual Communication Undergraduate Degree include the subject of Fundamentals of Radio and Television. In this context, it is relevant to analyse program offering through television and radio, and specially its link with media convergence. The knowledge and skills acquired by students of these degree in the field of most consumed media programm affects more comprehensive training and better preparation for later both professional and/or academic work. Television and radio are set on a central position into communication system, mainly due to the accumulation of audience and its consequent impact on the public sphere. In this sense, both media require careful study to cross its media nature, its program strategies and its development thorugh convergence. Moreover, it is essential to understand the mechanism and formas taken by the actors involved, wether entities operation nationwide or worldwide, to know their place into convergent context.
\end{abstract}

KEY WORDS: Program - Radio - Television - Convergence - Degree

\section{INTRODUCCIÓN}

\subsection{La convergencia mediática y la programación de contenidos}

La docencia en el ámbito de la programación de contenidos radiofónicos y televisivos se enmarca dentro de la materia "Fundamentos de la radio y la televisión". En el contexto de los planes de estudio de Grado en Comunicación Audiovisual de la Universitat Jaume I de Castellón, esta materia se desglosa en: la asignatura "Teoría y técnica de la radio y la televisión", impartida en segundo semestre del segundo curso académico; y la asignatura "Programación de radio y televisión", impartida en el primer semestre del tercer curso.

Los temarios de ambas asignaturas se encuentran coordinados en las vertientes teórica y práctica, para las cuales reservan tres créditos ECTS para cada una, hasta un total de seis créditos europeos para cada asignatura. De esta forma, los conocimientos y competencias que deben ser adquiridas por el alumnado en esta materia se inician en el segundo curso, para ser completadas con la asignatura de Programación de radio y televisión. 
En el actual contexto de convergencia, esta asignatura plantea la oferta mediática de contenidos radiofónicos y televisivos desde una perspectiva histórica aplicada a la evolución de la actividad profesional del programador de contenidos y al desarrollo de los medios comunicativos relacionados (radio y televisión); para situar ambos en el escenario mediático convergente.

El sistema comunicativo, ahora convergente, desempeña un papel fundamental en la esfera pública central, lo que recibe la atención de perspectivas teóricas como la Economía Política de la Comunicación (Mosco, 2009); (Miège, 2004); (Murdock \& Golding, 2005); (Castells, 2006). En este sentido, el conjunto de medios comunicativos, abanderados con la televisión, desarrollan una actividad protagonista en las sociedades avanzadas. La dualidad del sistema mediático, centrada en su carácter de industria cultural, combina la naturaleza creativas e industrial (Zallo, 2007), dota a sus contenidos de responsabilidad para la configuración de la sociedad de la información que, en el escenario convergente, se convierte en la sociedad red (Castells, 2001).

La función del programador de contenidos se perfila como fundamental, ya que estos ofrecen una considerable influencia política y social (Reig, 2009), especialmente en lo que se refiere a la formación de la opinión pública y la construcción de la realidad social (Casero, 2008). Esta labor se conjuga con la condición económica de los medios comunicativos de gestión privada, cuya búsqueda por la rentabilidad económica de su actividad marca las pautas de la oferta y la demanda de sus contenidos. En este aspecto, la publicidad adquiere un protagonismo relevante, que en ocasiones dificulta la consecución de los objetivos adheridos a la naturaleza simbólica del medio.

Además de la vertiente económica de los medios de comunicación, estos también se encuentran estrechamente ligados a la tecnología que les da soporte. El programador de contenidos se encuentra en el momento actual en una etapa de decisiones fundamentales en este aspecto, ya que no sólo debe planificar la oferta en función del medio en un sentido divergente, sino que debe responder a las demandas sociales vinculadas con el consumo convergente de 360 gados. En este sentido, los contenidos televisivos trascienden la pantalla del televisor, lo que dificulta el proceso de programación.

A pesar del escenario convergente, la televisión continúa desempeñando el papel de medio de comunicación estrella del conjunto mediático, vinculada estrechamente a la radio. Ambos son piezas fundamentales en el estudio de la comunicación, por lo que requieren un análisis en profundidad de su evolución y las tendencias de futuro desarrollo, considerando las adaptaciones a nuevos contextos. Para ello, resulta imprescindible conocer los mecanismos y las formas que adoptan los principales actores involucrados, ya sean entes que operan en el mercado doméstico como aquellos que operan en otros mercados, como el estadounidense, cuyas pautas se 
El escenario convergente presenta una evidente tendencia por compilar los contenidos audiovisuales en una pantalla única, que prolifera en diferentes soportes. Ordenador, Televisión con acceso a Internet, Tableta, Smartphone, etc. El consumo se individualiza y personaliza hasta cotas antes no alcanzables, con el video bajo demanda a través de Internet (iVOD). En este contexto, es imprescindible estudiar las pautas de actuación de los programadores de contenidos y su función de ordenación de la oferta, que permite observar cómo se construye el flujo programático en el medio divergente, para poder deconstruir la parrilla para su consumo unitario. En el especio convergente, la función del programador adquiere nuevas dimensiones, marcadas por la multidisciplinariedad, la cohesión de habilidad y estrategias transversales y los diseños programáticos de 360 grados.

\section{DESARROLLO}

\subsection{Objetivos y competencias del estudio de la programación mediática}

Los objetivos que se plantea el programa docente de la asignatura de "Programación de radio y televisión" son:

a) Ofrecer un estudio detallado de las técnicas y estrategias que influyen en la configuración de la programación de radio y televisión.

b) Evaluar la situación actual del mercado audiovisual español en materia de programación audiovisual.

c) Introducir al alumnado en los principales métodos de análisis de contenidos y los programas del medio radiofónico y televisivo.

d) Dotar al alumnado de capacidad de reflexión crítica sobre los contenidos y las últimas tendencias en programación audiovisual

Para la obtención de estos objetivos se han diseñado una serie de competencias que debe adquirir el alumnado. Las competencias se dividen en siete áreas de actividades: enseñanzas teóricas, enseñanzas prácticas, seminarios, tutorías, evaluación, trabajo personal y trabajo para la preparación de exámenes. 
Tabla 1. Objetivos por competencias

\begin{tabular}{|c|c|}
\hline Actividades & Competencias \\
\hline \multirow[t]{2}{*}{ Enseñanzas teóricas } & $\begin{array}{l}\text { - Capacidad para comprender el funcionamiento de las } \\
\text { técnicas y estrategias de programación audiovisual en los } \\
\text { medios radiofónico y televisivo }\end{array}$ \\
\hline & $\begin{array}{l}\text { - Competencia para exponer una reflexión crítica y un } \\
\text { análisis sobre las tendencias actuales de programación en } \\
\text { radio y tv, desde un estudio teórico y de campo } \\
\text { - Capacidad de análisis y síntesis } \\
\text { - Motivación por la calidad } \\
\text { - Razonamiento crítico }\end{array}$ \\
\hline $\begin{array}{l}\text { Enseñanzas } \\
\text { prácticas } \\
\text { (problemas) }\end{array}$ & $\begin{array}{l}\text { - Capacidad para el manejo de las principales metodologías } \\
\text { de análisis de programas y contenidos de las parrillas de } \\
\text { programación } \\
\text { - Capacidad para llevar a cabo diversas funciones relativas a } \\
\text { la elaboración de parrillas de programación } \\
\text { - Aprendizaje autónomo } \\
\text { - Capacidad de análisis y síntesis } \\
\text { - Capacidad de organización y planificación } \\
\text { - Creatividad } \\
\text { - Motivación por la calidad } \\
\text { - Razonamiento crítico } \\
\text { - Resolución de problemas } \\
\text { - Trabajo en equipo }\end{array}$ \\
\hline Seminarios & $\begin{array}{l}\text { - Competencia para el análisis de la situación actual del } \\
\text { mercado audiovisual español en materia de programación } \\
\text { - Razonamiento crítico } \\
\text { - Resolución de problemas }\end{array}$ \\
\hline Tutorías & $\begin{array}{l}\text { - Aprendizaje autónomo } \\
\text { - Motivación por la calidad }\end{array}$ \\
\hline Evaluación & - Todas las de la materia \\
\hline Trabajo personal & $\begin{array}{l}\text { - Capacidad para el manejo de las principales metodologías } \\
\text { de análisis de programas y contenidos de las parrillas de }\end{array}$ \\
\hline
\end{tabular}




\begin{tabular}{|l|l|}
\hline & programación \\
& $\begin{array}{l}\text { - Capacidad para llevar a cabo diversas funciones relativas a } \\
\text { la elaboración de parrillas de programación } \\
\text { - Competencia para el análisis de la situación actual del } \\
\text { mercado audiovisual español en materia de programación } \\
\text { - Aprendizaje autónomo }\end{array}$ \\
\hline $\begin{array}{l}\text { Trabajo de } \\
\text { preparación de los } \\
\text { exámenes }\end{array}$ & $\begin{array}{l}\text { - Aprendizaje autónomo } \\
\text { - Capacidad de gestión de la información }\end{array}$ \\
\hline
\end{tabular}

Cada una de estas actividades tiene una estimación en horas presenciales o no presenciales para la consecución de las competencias correspondientes. En este aspecto, para el grupo de enseñanzas teóricos se calcula un total de 24 horas presenciales en el aula. La misma cantidad se calcula para las enseñanzas prácticas. Por su parte, las tutorías y la evaluación estiman un total de 3 horas presenciales para cada una de estas actividades, mientras que el seminario consta de 6 horas presenciales.

Por otra parte, el trabajo personal del alumnado para la adquisición de las competencias marcadas ronda las 54 horas de trabajo autónomo, mientras que la preparación de los exámenes se estima en un total de 36 horas repartidas a lo largo del semestre.

La suma de esta dedicación por parte del alumnado alcanza las 60 horas presenciales y las 90 horas no presenciales, con un total de 150 horas, correspondientes a los 6 créditos ECTS que tiene la asignatura.

\subsection{Metodología docente}

La asignatura parte de una metodología que separa entre teoría y práctica, que se desarrollan de forma alternada. La teoría se basa en exposiciones de temas teóricos por parte del docente, que se complementa con ejercicios y ejemplos relacionados con los conocimientos impartidos. En la fase práctica se estimula la asimilación de conceptos a través del trabajo individual y grupal del alumnado, que se relacionan con los temas principales de la materia. Las sesiones prácticas ocupan 4 horas semanales ( 2 horas teoría y 2 horas práctica). 
El temario teórico se encuentra estructurado en 7 temas, de la siguiente manera:

a) Tema 1. El concepto de programación: 1.1. Definición y caracterización; 1.2. Elementos implicados en la programación; 1.3. Modelos de programación

b) - Tema 2. Historia de la programación: 2.1. Antecedentes históricos de la programación; 2.2. La programación en los entes públicos de radiotelevisión: la idea de servicio público; 2.3. Los inicios de la programación en España: el caso de RTVE; 2.4. La desregulación y las transformaciones de la programación; 2.5. El impacto de la televisión de pago en la concepción de la programación

c) Tema 3. Los públicos y la audiencia de la programación: 3.1. Tipología, perfiles y características; 3.2. El consumo de radio y televisión; 3.3. La investigación de audiencias

d) Tema 4. Técnicas y estrategias de programación: 4.1. Las franjas horaria y la distribución de los programas; 4.2. La construcción de la parrilla; 4.3. Coyunturas de competencia; 4.4. Técnicas de programación

e) Tema 5. La programación en radio y televisión dentro del mercado español actual: 5.1. Marco general; 5.2. Políticas de programación de los operadores de radio y televisión; 5.3. Seguimiento y evaluación de las últimas temporadas; 5.4. Tendencias actuales de la programación de contenidos.

f) Tema 6. El marco legal de la programación audiovisual: 6.1. Normativas españolas en materia de programación y contenidos; 6.2. La autorregulación de la programación audiovisual

g) Tema 7. El futuro de la programación en el escenario digital: 7.1. Transformaciones del contexto de consumo y cambios en las audiencias; 7.2. Nuevo escenario digital; 7.3. ¿Hacia el fin de la programación?

Respecto a la organización de las actividades prácticas, en el inicio del semestre se divide el conjunto global de alumnado en dos grupos (L1 y L2), de 45 alumnos cada uno, para su posterior subdivisión en grupos de no más de 8 personas, para trabajar las propuestas prácticas grupales.

La asignatura se complementa con un seminario, cuya asistencia por parte del alumnado es obligatoria. El alumnado deberá preparar de forma individual una reflexión y/o análisis relacionado con la temática expuesta durante el seminario, en función de las directrices que se le otorguen.

El temario teórico se encuentra completado con el temario práctico, que engloba las siguientes propuestas:

P1. El debate sobre la calidad de la programación: la emergencia de la "telebasura" P2. Los contenidos de la programación radiofónica P3. El mercado de la ficción P4. La programación infantil P5. La creación de programas y formatos televisivos P6. La elaboración de parrillas de programación. La metodología de la asignatura se apoya 
fundamental para el seguimiento de las sesiones teóricas y la preparación del trabajo autónomo del estudiantado, y bibliografía de consulta complementaria. En este caso, se ofrece un listado amplio de referencias enfocadas a la profundización de los conceptos vistos en las sesiones presenciales en función de las necesidades específicas de las tareas prácticas a realizar, pero también en función de los intereses más particulares que pueda suscitar el temario en cada estudiante.

Entre los títulos básicos más destacados se encuentran los de: Álvarez Monzoncillo (2011), Blum y Lindheim (Blum \& Lindheim, 1989), Castells (Castells, 2006), Contreras y Palacio (Contreras \& Palacio, 2003), Cortés (Cortés, 1999), Martí (Martí, 2000), Wolf (Wolf, 1989) y Zallo (Zallo, 2007).

Como se ha señalado, la asignatura combina sesiones presenciales, trabajo en laboratorio y trabajo autónomo por parte del alumnado. Éste deberá cumplir con todas las fases para superar la asignatura.

\subsection{Sistema de evaluación}

La evaluación de la asignatura se divide entre la teoría y la práctica. Es imprescindible superar cada una de esas partes con al menos un 5 sobre 10 puntos. La evaluación se realiza sobre una serie de pruebas. $\mathrm{Al}$ final del semestre se realizará un examen escrito $(40 \%)$, en las fechas de las convocatorias oficiales. El examen evaluará los conocimientos adquiridos por el alumnado, su capacidad para aplicarlos y para razonar de forma crítica. Los ejercicios y ejemplos desarrollados y planteados durante las sesiones teóricas persiguen la adquisición de estas competencias por parte del alumnado. El resto de pruebas para la evaluación son: la presentación oral de trabajos y pósters $(10 \%)$ y la presentación de proyectos $(20 \%)$, la resolución de casos $(15 \%)$ y la observación/ejecución de las tareas y prácticas (5\%). 
Tabla 2. Sistema de evaluación

\begin{tabular}{|l|l|}
\hline Prueba de evaluación & Competencias \\
\hline
\end{tabular}

\begin{tabular}{|c|c|}
\hline $\begin{array}{l}\text { Elaboración de trabajos } \\
\text { académicos }\end{array}$ & $\begin{array}{l}\text { Capacidad para el manejo de las principales } \\
\text { metodologías de análisis de programas y contenidos } \\
\text { de las parrillas de programación } \\
\text { Creatividad }\end{array}$ \\
\hline $\begin{array}{l}\text { Examen escrito (test, } \\
\text { desarrollo y/o problemas) }\end{array}$ & $\begin{array}{l}\text { Capacidad para comprender el funcionamiento de las } \\
\text { técnicas y estrategias de programación audiovisual en } \\
\text { los medios radiofónico y televisivo } \\
\text { Competencia para exponer una reflexión crítica y un } \\
\text { análisis sobre las tendencias actuales de programación } \\
\text { en radio y TV, desde un estudio teórico y de campo } \\
\text { Aprendizaje autónomo } \\
\text { Capacidad de análisis y síntesis } \\
\text { Capacidad de gestión de la información } \\
\text { Motivación por la calidad } \\
\text { Razonamiento crítico }\end{array}$ \\
\hline $\begin{array}{l}\text { Observación/ejecución de } \\
\text { tareas y prácticas }\end{array}$ & $\begin{array}{l}\text { Capacidad de organización y planificación } \\
\text { Trabajo en equipo }\end{array}$ \\
\hline $\begin{array}{l}\text { Presentaciones orales } y \\
\text { pósters }\end{array}$ & $\begin{array}{l}\text { Competencia para el análisis de la situación actual del } \\
\text { mercado audiovisual español en materia de } \\
\text { programación } \\
\text { Capacidad de análisis y síntesis } \\
\text { Trabajo en equipo }\end{array}$ \\
\hline Proy & $\begin{array}{l}\text { Capacidad para llevar a cabo diversas funciones } \\
\text { relativas a la elaboración de parrillas de programación } \\
\text { Creatividad } \\
\text { Motivación por la calidad } \\
\text { Trabajo en equipo }\end{array}$ \\
\hline Resolución de Casos & I funcionamiento de las \\
\hline
\end{tabular}




\begin{tabular}{|l|l|}
\hline técnicas y estrategias de programación audiovisual en \\
los medios radiofónico y televisivo \\
Resolución de problemas \\
Trabajo en equipo
\end{tabular}

Si por algún motivo, debidamente justificado y documentado, parte del alumnado no pudiera asistir de forma presencial a las sesiones prácticas se le aplica un itinerario alternativo. En ese caso, el alumnado debe asumir la responsabilidad de desarrollar un programa práctico de laboratorio fuera de las sesiones impartidas por el docente, que le dedicará las horas de tutorías estipuladas para cada alumno/a del curso.

Para superar la asignatura, el alumnado deberá asistir a las sesiones teóricas, al seminario y a las sesiones prácticas. Asimismo, deberá entregar los ejercicios y ejemplos planteados y las prácticas programadas cumpliendo los plazos establecidos en el cronograma de la asignatura.

\section{CONCLUSIONES}

La asignatura "Programación de radio y televisión" presenta un programa docente diseñado para ubicar el estudio en el contexto de convergencia mediática en el que se encuentran los medios de comunicación, marcado por la multiplicidad de la oferta, la dispersión de las audiencias y el consumo personalizado y social.

La asignatura plantea el concepto de programación y su análisis a través de los medios audiovisuales y su evolución histórica. Se presta atención al estudio crítico de conceptos teóricos, así como a la praxis de la programación radiofónica y televisiva. Se abordan las técnicas y estrategias que se aplican en la programación de contenidos, a partir del análisis del mercado audiovisual actual, tanto en el contexto español, como en el contexto internacional. Finalmente, motiva la reflexión y el análisis sobre las tendencias de programación de contenidos en el escenario convergente.

A pesar de la relevancia de estas competencias para el estudiante de Comunicación Audiovisual, se detecta una escasa presencia de esta asignatura en los planes de estudios de las facultades de Ciencias de la Información (Contreras y Palacio, 2003). Su estudio pretende dotar al alumnado de conocimientos, habilidades y competencias para la ejecución de la función del programador de contenidos, pero también para la profundización en el conocimiento de los medios de comunicación como ofertares de contenidos con una fuerte vinculación con la sociedad, lo que repercute en el mejor entendimiento de otras facetas de la profesión comunicativa, como la creación de esos contenidos. 


\section{REFERENCIAS}

Álvarez Monzoncillo, J. M. (2011). La televisión etiquetada: nuevas audiencias, nuevos negocios. Madrid: Ariel.

Blum, R. A. \& Lindheim, R. D. (1989). Programación de las cadenas de televisión en horario de máxima audiencia. Madrid: IORTV.

Casero, A. (2008). La construcción mediática de las crisis politicas. Madrid: Fragua.

Castells, M. (2006). La sociedad red: una visión global. Madrid: Alianza.

Castells, M. (2001). La galaxia Internet. Barcelona: Plaza\&Janés.

Contreras, J. M. \& Palacio, M. (2003). La programación de televisión. Madrid: Síntesis.

Cortés, J. Á. (1999). La estrategia de la seducción. La programación en la neotelevisión. Pamplona: Eunsa.

GECA. (1998). El anuario de la televisión. Madrid: GECA. Ediciones anuales desde 1998 hasta la actualidad.

Jauset, J. A. (2000). La investigación de audiencias en televisión. Barcelona: Paidós.

Martí, J. M. (2000). De la idea a l'antena. Tècniques de programació radiofónica.

Barcelona: Pòrtic.

Mosco, V. (2009). The Political Economy of Communication. London: Sage.

Murdock, G. \& Golding, P. (2005). Culture, Communications and Political Economy. En Mass Media and Society. London: Hodder Arnold.

Reig, R. (2009). Bases teóricas y documentales para el estudio de la Estructura de la Información y el análisis estructural de los mensajes. Revista Estudios sobre el mensaje periodístico, 15 .

Zallo, R. (2007). La economía de la cultura (y de la comunicación) como objeto de estudio. Revista Zer, 22.

Wolf, M. (1989). Géneros y televisión. Revista Anàlisi: quaderns de comunicación i cultura, 9 . 


\section{Jéssica Izquierdo-Castillo}

Profesora del Departamento de Ciencias de la Comunicación, en la Universidad Jaume I de Castellón. Es Doctora en Comunicación Audiovisual y cursó las licenciaturas de Publicidad y Relaciones Públicas y de Comunicación Audiovisual (Universidad de Valencia). Entre las líneas de investigación que desarrolla destaca el ámbito de la estructura del sistema comunicativo, especialmente de los sectores de cine, televisión y prensa. Así como sobre las políticas de comunicación; así como las políticas de comunicación. 